\title{
Parametric estimation for the standard and geometric telegraph process observed at discrete times
}

\author{
Alessandro De Gregorio • Stefano Maria Iacus
}

Received: 12 September 2006 / Accepted: 19 July 2007 / Published online: 11 December 2007

(C) Springer Science+Business Media B.V. 2007

\begin{abstract}
The telegraph process $X(t), t \geq 0$, (Goldstein, Q J Mech Appl Math 4:129-156, $1951)$ and the geometric telegraph process $S(t)=s_{0} \exp \left\{\left(\mu-\frac{1}{2} \sigma^{2}\right) t+\sigma X(t)\right\}$ with $\mu$ a known real constant and $\sigma>0$ a parameter are supposed to be observed at $n+1$ equidistant time points $t_{i}=i \Delta_{n}, i=0,1, \ldots, n$. For both models $\lambda$, the underlying rate of the Poisson process, is a parameter to be estimated. In the geometric case, also $\sigma>0$ has to be estimated. We propose different estimators of the parameters and we investigate their performance under the asymptotics, i.e. $\Delta_{n} \rightarrow 0, n \Delta_{n}=T<\infty$ as $n \rightarrow \infty$, with $T>0$ fixed. The process $X(t)$ in non markovian, non stationary and not ergodic thus we build a contrast function to derive an estimator. Given the complexity of the equations involved only estimators on the first model can be studied analytically. Therefore, we run an extensive Monte Carlo analysis to study the performance of the proposed estimators also for small sample size $n$.
\end{abstract}

Keywords Telegraph process · Discretely observed process ·

Inference for stochastic processes

Mathematics Subject Classification (2000) Primary 60K99 · Secondary 62M99

\section{Introduction}

The random motions with finite velocity represent an alternative to diffusion models defined by means of stochastic differential equations. The prototype of these models is the telegraph process (see Goldstein (1951); Kac (1974)) that describes the position of a particle moving on the real line, alternatively with constant velocity $+v$ or $-v$. The changes of direction are

\footnotetext{
A. De Gregorio $(\bowtie) \cdot$ S. M. Iacus

Department of Economics, Business and Statistics, University of Milan,

Via Conservatorio 7, 20122 Milan, Italy

e-mail: alessandro.degregorio@unimi.it

S. M. Iacus

e-mail: stefano.iacus@unimi.it
} 
governed by an homogeneous Poisson process $N(t)$ with rate $\lambda>0$. The telegraph process ${ }^{1}$ is defined by

$$
X(t)=V(0) \int_{0}^{t}(-1)^{N(s)} \mathrm{d} s, \quad t>0,
$$

where the initial velocity $V(0)$ assumes the values $\pm v$ with equal probability and independently of $N(t)$. We consider a particle initially located on the real line at point $x_{0}$.

The explicit conditional density function of the process $X(t)$ has been obtained by Orsingher (1990) and reads

$$
\begin{aligned}
p\left(x, t ; x_{0}, 0\right)= & \frac{e^{-\lambda t}}{2 v}\left\{\lambda I_{0}\left(\frac{\lambda}{v} \sqrt{v^{2} t^{2}-\left(x-x_{0}\right)^{2}}\right)\right. \\
& \left.+\frac{\partial}{\partial t} I_{0}\left(\frac{\lambda}{v} \sqrt{v^{2} t^{2}-\left(x-x_{0}\right)^{2}}\right)\right\} \chi_{\left\{\left|x-x_{0}\right|<v t\right\}} \\
& +\frac{e^{-\lambda t}}{2}\left\{\delta\left(x-x_{0}-v t\right)+\delta\left(x-x_{0}+v t\right)\right\} \\
= & \frac{e^{-\lambda t}}{2 v}\left\{\lambda I_{0}\left(\frac{\lambda}{v} \sqrt{v^{2} t^{2}-\left(x-x_{0}\right)^{2}}\right)\right. \\
& \left.+\frac{v \lambda t I_{1}\left(\frac{\lambda}{v} \sqrt{v^{2} t^{2}-\left(x-x_{0}\right)^{2}}\right)}{\sqrt{v^{2} t^{2}-\left(x-x_{0}\right)^{2}}}\right\} \chi_{\left\{\left|x-x_{0}\right|<v t\right\}} \\
& +\frac{e^{-\lambda t}}{2}\left\{\delta\left(x-x_{0}-v t\right)+\delta\left(x-x_{0}+v t\right)\right\} \\
= & \frac{e^{-\lambda t}}{2 v}\left\{\lambda I_{0}\left(\frac{\lambda}{v} \sqrt{u_{t}\left(x, x_{0}\right)}\right)\right. \\
& \left.+\frac{v \lambda t I_{1}\left(\frac{\lambda}{v} \sqrt{u_{t}\left(x, x_{0}\right)}\right)}{\sqrt{u_{t}\left(x, x_{0}\right)}}\right\} \chi_{\left\{u_{t}\left(x, x_{0}\right)>0\right\}} \\
& +\frac{e^{-\lambda t}}{2} \delta\left(u_{t}\left(x, x_{0}\right)\right)
\end{aligned}
$$

where $x$ is such that $\left|x-x_{0}\right| \leq v t, u_{t}\left(x, x_{0}\right)=v^{2} t^{2}-\left(x-x_{0}\right)^{2}, \chi(\cdot), \delta(\cdot)$ and

$$
I_{\nu}(x)=\sum_{k=0}^{\infty}\left(\frac{x}{2}\right)^{2 k+v} \frac{1}{k ! \Gamma(k+v+1)},|x|<\infty,|\arg x|<\pi, v \in R,
$$

is the modified Bessel function with imaginary argument. Note that the second term in Eq. 2 represents the singular component of the distribution of (1), of the position of the particle at time $t$. Indeed, if no Poisson events occur in the interval $[0, t]$, we have that $P\{X(t)=+v t\}=P\{X(t)=-v t\}=\frac{1}{2} e^{-\lambda t}$.

Many authors analyzed over the years the telegraph process, see for example Orsingher (1990, 1995), Foong and Kanno (1994), Stadje and Zacks (2004). Di Crescenzo and Pellerey (2002) proposed the geometric telegraph process as a model to describe the dynamics of the price of risky assets $S(t)$. In the Black-Scholes (1973) - Merton (1973) model the process

\footnotetext{
${ }^{1}$ In the literature, this process is alternatively called the telegraph process or the telegrapher's process.
} 
$S(t)$ is described by means of geometric Brownian motion

$$
S(t)=s_{0} \exp \{\alpha t+\sigma W(t)\}, \quad t \geq 0 .
$$

$S(0)=s_{0}$, where $W(t)$ is a standard Brownian motion and $\alpha=\mu-\frac{1}{2} \sigma^{2}$, with $\mu \in R$, $\sigma>0$. Di Crescenzo and Pellerey (2002) assume that $S(t)$ evolves in time according to the following process

$$
S(t)=s_{0} \exp \{\alpha t+\sigma X(t)\}, \quad t \geq 0 .
$$

where $X(t)$ is the telegraph process. Given that $X(t)$ has bounded variation, so is $S(t)$ in Eq. 4. This seems a realistic way to model paths of assets in the financial markets. Mazza and Rulliére (2004) linked the process (1) and the ruin processes in the context of risk theory. Di Masi et al. (1994) propose to model the volatility of financial markets in terms of the telegraph process. Ratanov $(2004,2005)$ propose to model financial markets using a telegraph process with two intensities $\lambda_{ \pm}$and two velocities $c_{ \pm}$. While such markets may admit an arbitrage opportunity, linking opportunity velocities and interest rates, the author proves that the market becomes arbitrage-free and complete. An analogous of the Black and Scholes equation is established as well.

The aim of this paper is the estimation of the parameter $\lambda$ when $\{X(t), 0 \leq t \leq T\}$ is observed at equidistant times $0=t_{0}<\cdots<t_{n}$ (and also $\sigma$ for discrete observations from the process (4)). We assume that $t_{i}=i \Delta_{n}, i=0, \ldots, n$, hence $n \Delta_{n}=T$. The asymptotic framework is the following: $n \Delta_{n}=T$ fixed and $\Delta_{n} \rightarrow 0$ as $n \rightarrow \infty$. When the telegraph process $X(t)$ is observed continuously then $N(T) / T$ is the optimal estimator of the parameter $\lambda$ as this statistical problem is equivalent to the one of the observation of the whole Poisson process ${ }^{2}$ on $[0, T]$. This situation corresponds indeed to the limiting experiment in our asymptotic framework. This asymptotic framework is a well known scheme in the literature on estimation from discrete time observations of processes solution to the following stochastic differential equation

$$
\mathrm{d} Y(t)=b(Y(t), \theta) \mathrm{d} t+\sigma(Y(t), \theta) \mathrm{d} W(t) .
$$

This field has been an active research area during the last twenty years. The reader can consult Sørensen (2004) for a review on estimation techniques recently appeared in the literature since the seminal papers of Le Breton (1976) and Florens-Zmirou (1989): e.g. estimating functions, analytical and numerical approximations of the likelihood function, MCMC methods, indirect inference, etc. Unfortunately such methods are not directly applicable in our case because the telegraph process is not ergodic or stationary nor markovian. The main idea in the paper is to consider the observed increments of the process $X\left(i \Delta_{n}\right)-X\left((i-1) \Delta_{n}\right)$ as $n$ copies of the telegraph process up to time $\Delta_{n}$ and treat them as if they were independent (which is untrue). From this idea we build a contrast function from which we derive an estimator. We further propose a moment type estimator based on the second moment of the telegraph process. Equations emerging in connection with the telegraph process are always complicated to treat and closed form results are quite rare in the literature. This also happens in our work, thus in some cases we rely on numerical simulations to study the properties of the proposed estimators.

It is worth mention that, up to our knowledge, the only references about estimation problems for the telegrapher's processes are Yao (1985) and Iacus (2001). The first author considers the problem of state estimation of the telegrapher's process under white noise perturbation and studies performance of nonlinear filters. The second paper is about the estimation of the

${ }^{2}$ For more details on parametric estimation for Poisson process see Kutoyants (1998). 
parameter $\theta$ of the non-constant rate $\lambda_{\theta}(t)$ from continuous observations of the process. Very recently, Iacus and Yoshida (2006) consider $^{3}$ estimation problem for this process under the large sample asymptotic scheme, i.e. $T=n \Delta_{n} \rightarrow \infty$ and $n \Delta_{n}^{3} \rightarrow 0$ as $n \rightarrow \infty$, but their approach is based on different arguments.

The paper is organized as follows. In Sect. 2 we will introduce the following contrast function which plays the role of a pseudo-likelihood function

$$
L_{n}(\lambda)=\prod_{i=1}^{n} p\left(X_{i}, \Delta_{n} ; X_{i-1}, t_{i-1}\right)
$$

where $p\left(X_{i}, \Delta_{n} ; X_{i-1}, t_{i-1}\right)$ is defined by (2). We will then study the the estimator $\hat{\lambda}_{n}$ satisfying

$$
\left.\frac{\partial}{\partial \lambda} \log L_{n}(\lambda)\right|_{\lambda=\hat{\lambda}_{n}}=0
$$

and then show that this estimator is also the unique solution of

$$
\hat{\lambda}_{n}=\arg \max _{\lambda>0} L_{n}(\lambda)
$$

which is interpreted as a pseudo-maximum likelihood estimator. We also propose a moment type estimator for $\lambda$ based on the second moment of the process $X(t)$. Inference problems and estimators for the parameters $\lambda$ and $\sigma$ of the geometric telegrapher's process are considered in Sect. 3. Finally, Sect. 4 contains a Monte Carlo analysis to study empirically the behavior of the estimators in a finite sample context (i.e. non asymptotically).

\section{Inference for the telegraph process}

We assume that the telegraph process $\{X(t), 0 \leq t \leq T\}$, with $X(0)=x_{0}=0$, is observed only at equidistant discrete times $0<t_{1}<\cdots<t_{n}=T$, with $t_{i}=i \Delta_{n}, i=0, \ldots, n$ (hence $\left.n \Delta_{n}=T\right)$. We use the following notation to simplify the formulas: $X\left(t_{i}\right)=X\left(i \Delta_{n}\right)=X_{i}$. The asymptotic is considered as $n$ tends to infinity under the conditions $\Delta_{n} \rightarrow 0$ and $n \Delta_{n}=T$. The interest is in the estimation of the parameter $\lambda$ whilst $v$ is assumed to be known.

As mentioned in the Introduction, if one can observe the whole trajectory, $\lambda$ can be estimated as $N(T) / T$ where $N(T)$ is the number of times the process switches its velocity during the interval $[0, T]$ which is, of course, the number of Poisson events counted in $[0, T]$. This is certainly the best estimator of $\lambda$ and it is indeed our target.

The estimation of $v$ is always an uninteresting problem. In fact, if there are no switchings in $\left[(i-1) \Delta_{n}, i \Delta_{n}\right]$ then $X_{i}-X_{i-1}=v \Delta_{n}$, hence if $\Delta_{n}$ is sufficiently small, there is high probability of observing $N\left(t_{i+1}\right)-N\left(t_{i}\right)=0$ and $v$ can be estimated (actually calculated) without error.

The process $X(t)$ itself is not markovian, whilst the two dimensional process $(X(t), V(t))$ (where $V(t)=V(0)(-1)^{N(t)}$ ) has the Markov property. However, a scheme of observation in which one is able to observe both the position and the velocity of the process at discrete time instants is not admissible, so we can rely only on the observation of the $X(t)$ component. Hence we cannot write an explicit likelihood of the process in the form of a product of transition densities as, for example, for the case of diffusion processes. Another unfortunate fact about the telegraph process is that it is not even stationary (at the second order) as

$$
E X(t)=0
$$

\footnotetext{
3 The cited reference chronologically follows the present work.
} 
and

$$
E X^{2}(t)=\frac{v^{2}}{\lambda}\left(t-\frac{1-e^{-2 \lambda t}}{2 \lambda}\right)
$$

(see Orsingher (1990)) nor it posseses an ergodic property, so we cannot use the same approach proposed, e.g., in Sørensen (2000).

\subsection{The pseudo-likelihood function}

By taking into account the distribution (2), we build a contrast function (which is a pseudolikelihood function of the process) as follows

$$
\begin{aligned}
L_{n}(\lambda)= & L_{n}\left(\lambda \mid X_{0}, X_{1}, \ldots, X_{n}\right)=\prod_{i=1}^{n} p\left(X_{i}, \Delta_{n} ; X_{i-1}, t_{i-1}\right) \\
= & \prod_{i=1}^{n}\left\{\frac{e^{-\lambda \Delta_{n}}}{2 v}\left\{\lambda I_{0}\left(\frac{\lambda}{v} \sqrt{u_{n, i}}\right)+\frac{v \lambda \Delta_{n} I_{1}\left(\frac{\lambda}{v} \sqrt{u_{n, i}}\right)}{\sqrt{u_{n, i}}}\right\} \chi_{\left\{u_{n, i}>0\right\}}\right. \\
& \left.+\frac{e^{-\lambda \Delta_{n}}}{2} \delta\left(u_{n, i}=0\right)\right\}
\end{aligned}
$$

where $u_{n, i}=u_{n}\left(X_{i}, X_{i-1}\right)=v^{2} \Delta_{n}^{2}-\left(X_{i}-X_{i-1}\right)^{2}$.

The density $p\left(X_{i}, \Delta_{n} ; X_{i-1}, t_{i-1}\right)$ appearing in (8) is the probability law of a telegraph process initially located in $X_{i-1}$, that reaches the position $X_{i}$ at time $t_{i}$. The construction of $L_{n}(\lambda)$ is based on the following assumption: the observed increments $X_{i}-X_{i-1}$ are $n$ copies of the process $X\left(\Delta_{n}\right)$ (i.e. the process $X(t)$ up to time $\left.\Delta_{n}\right)$ and treated as if they were independent. This is of course untrue, but the estimators based on $L_{n}(\lambda)$ posses reasonable properties. It is clear that (8) is equivalent to

$$
\begin{aligned}
L_{n}(\lambda)= & \left(\frac{e^{-\lambda \Delta_{n}}}{2}\right)^{n-n^{+}} \\
& \times \prod_{i=1}^{n^{+}} \frac{e^{-\lambda \Delta_{n}}}{2 v}\left\{\lambda I_{0}\left(\frac{\lambda}{v} \sqrt{u_{n, i}}\right)+\frac{v \lambda \Delta_{n} I_{1}\left(\frac{\lambda}{v} \sqrt{u_{n, i}}\right)}{\sqrt{u_{n, i}}}\right\} \\
= & \frac{e^{-\lambda n \Delta_{n}}}{2^{n}} \frac{1}{v^{n^{+}}} \prod_{i=1}^{n^{+}}\left\{\lambda I_{0}\left(\frac{\lambda}{v} \sqrt{u_{n, i}}\right)+\frac{v \lambda \Delta_{n} I_{1}\left(\frac{\lambda}{v} \sqrt{u_{n, i}}\right)}{\sqrt{u_{n, i}}}\right\}
\end{aligned}
$$

where $n^{+}$is equal to the number of telegraph processes $X\left(\Delta_{n}\right)$ with at least one change of direction (see Fig. 1).

In the expression (9), the factor $\left(\frac{e^{-\lambda \Delta_{n}}}{2}\right)^{n-n^{+}}$concerns the singular part of the densities $p\left(X_{i}, \Delta_{n} ; X_{i-1}, t_{i-1}\right)$, while the product represents the absolutely continuous component of the distributions of the telegraph processes. Note that for increasing values of $\lambda$, the absolutely continuous component of (9) has a bigger weight than the discrete component; viceversa for small values of $\lambda$. This has consequences in the performance of the estimators as shown in Sect. 4. Figure 1 shows how the two components of the function $L_{n}(\lambda)$ emerge for this scheme of observation. 


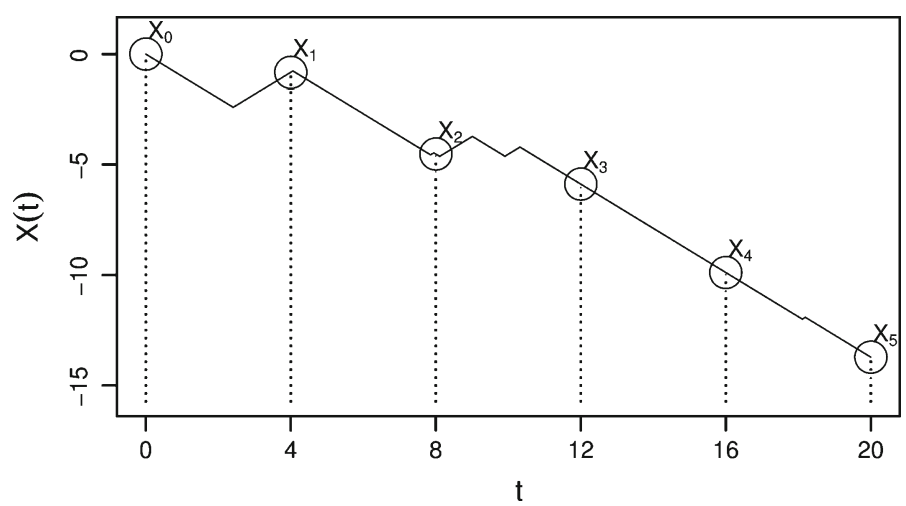

Fig. 1 Discrete time sampling of the telegraph process. Between $X_{3}$ and $X_{4}$ no Poisson event occurred with probability $e^{-\lambda \Delta_{n}}$, hence the singular part of the approximated likelihood emerges in the $L_{n}(\lambda)$. For this example trajectory $n=5$ and $n^{+}=4$

\subsection{Minimum contrast estimator}

Given the following contrast function

$$
F\left(\lambda ; X_{1}, \ldots, X_{n}\right)=\frac{\partial}{\partial \lambda} \log L_{n}(\lambda)
$$

we consider the estimator $\hat{\lambda}_{n}$ solution to $F(\lambda)=0$, i.e.

$$
\hat{\lambda}_{n}: F\left(\lambda=\hat{\lambda}_{n} ; X_{1}, \ldots, X_{n}\right)=0
$$

and next theorem proves the uniqueness of the solution of (11).

Theorem 2.1 The estimator $\hat{\lambda}_{n}$ in (11) is unique.

Proof To prove the result, we show that the second partial derivative of $\log L_{n}(\lambda)$ is negative. We start by writing

$$
\begin{aligned}
\log L_{n}(\lambda)=- & \lambda n \Delta_{n}-\log \left(2^{n} v^{n^{+}}\right) \\
& +\sum_{i=1}^{n^{+}} \log \left\{\lambda I_{0}\left(\frac{\lambda}{v} \sqrt{u_{n, i}}\right)+\frac{v \lambda \Delta_{n} I_{1}\left(\frac{\lambda}{v} \sqrt{u_{n, i}}\right)}{\sqrt{u_{n, i}}}\right\}
\end{aligned}
$$

and direct differentiation of (12) with respect to $\lambda$ gives

$$
\begin{aligned}
& -n \Delta_{n}+\sum_{i=1}^{n^{+}}\left\{\frac{I_{0}\left(\frac{\lambda}{v} \sqrt{u_{n, i}}\right)\left(1+\frac{\lambda \Delta_{n}}{2}\right)}{\lambda I_{0}\left(\frac{\lambda}{v} \sqrt{u_{n, i}}\right)+\frac{\lambda v \Delta_{n}}{\sqrt{u_{n, i}}} I_{1}\left(\frac{\lambda}{v} \sqrt{u_{n, i}}\right)}\right. \\
& +\frac{\left(\frac{\lambda \sqrt{u_{n, i}}}{v}+\frac{v \Delta_{n}}{\sqrt{u_{n, i}}}\right) I_{1}\left(\frac{\lambda}{v} \sqrt{u_{n, i}}\right)}{\lambda I_{0}\left(\frac{\lambda}{v} \sqrt{u_{n, i}}\right)+\frac{\lambda v \Delta_{n}}{\sqrt{u_{n, i}}} I_{1}\left(\frac{\lambda}{v} \sqrt{u_{n, i}}\right)} \\
& \left.+\frac{\frac{\lambda \Delta_{n}}{2} I_{2}\left(\frac{\lambda}{v} \sqrt{u_{n, i}}\right)}{\lambda I_{0}\left(\frac{\lambda}{v} \sqrt{u_{n, i}}\right)+\frac{\lambda v \Delta_{n}}{\sqrt{u_{n, i}}} I_{1}\left(\frac{\lambda}{v} \sqrt{u_{n, i}}\right)}\right\}
\end{aligned}
$$


In view of the following property of Bessels functions

$$
I_{\nu-1}(z)-I_{v+1}(z)=\frac{2 v}{z} I_{\nu}(z)
$$

we have that

$$
-\frac{\lambda \Delta_{n}}{2}\left\{I_{0}\left(\frac{\lambda}{v} \sqrt{u_{n, i}}\right)-I_{2}\left(\frac{\lambda}{v} \sqrt{u_{n, i}}\right)\right\}=-\frac{\Delta_{n} v}{\sqrt{u_{n, i}}} I_{1}\left(\frac{\lambda}{v} \sqrt{u_{n, i}}\right)
$$

therefore (13) can be rewritten as

$$
\begin{aligned}
\frac{\partial}{\partial \lambda} \log L_{n}(\lambda)= & -n \Delta_{n} \\
& +\sum_{i=1}^{n^{+}}\left\{\frac{\sqrt{u_{n, i}} v\left(1+\Delta_{n} \lambda\right) I_{0}\left(\frac{\lambda}{v} \sqrt{u_{n, i}}\right)}{v \lambda\left(\sqrt{u} I_{0}\left(\frac{\lambda}{v} \sqrt{u_{n, i}}\right)+v \Delta_{n} I_{1}\left(\frac{\lambda}{v} \sqrt{u_{n, i}}\right)\right)}\right. \\
& \left.+\frac{u_{n, i} \lambda I_{1}\left(\frac{\lambda}{v} \sqrt{u_{n, i}}\right)}{v \lambda\left(\sqrt{u} I_{0}\left(\frac{\lambda}{v} \sqrt{u_{n, i}}\right)+v \Delta_{n} I_{1}\left(\frac{\lambda}{v} \sqrt{u_{n, i}}\right)\right)}\right\}
\end{aligned}
$$

Differentiating (15) again with respect to $\lambda$ and posing $x=\frac{\lambda}{v} \sqrt{u_{n, i}}$ to simplify the equations ${ }^{4}$, we obtain, after tedious calculations, that

$$
\frac{\partial^{2}}{\partial \lambda^{2}} \log L_{n}(\lambda)=\sum_{i=1}^{n^{+}} \frac{g_{i}}{2 v^{2} \lambda^{2}\left(\sqrt{u_{n, i}} I_{0}(x)+v \Delta_{n} I_{1}(x)\right)^{2}}
$$

where the generic term $g_{i}$ is the sum of the following terms

$$
\begin{gathered}
-2 \sqrt{u_{n, i}} v^{3} \Delta_{n} I_{1}(x) I_{0}(x)<0, \\
u_{n, i}\left\{u_{n, i} \lambda^{2}-v^{2}\left(2+\Delta_{n} \lambda+\Delta_{n}^{2} \lambda^{2}\right)\right\} I_{0}^{2}(x), \\
2 u_{n, i} \lambda\left\{-\lambda u_{n, i}+v^{2} \Delta_{n}\left(1+\Delta_{n} \lambda\right)\right\} I_{1}^{2}(x), \\
u_{n, i} \lambda\left(u_{n, i} \lambda-v^{2} \Delta_{n}\left(1+\Delta_{n} \lambda\right)\right) \cdots I_{2}(x) I_{0}(x) .
\end{gathered}
$$

Recalling that $u_{n, i}=v^{2} \Delta_{n}^{2}-\left(X_{i}-X_{i-1}\right)^{2}>0$, Eq. 17 can be rewritten as

$$
-u_{n, i}\left\{v^{2} \Delta_{n} \lambda+\left(X_{i}-X_{i-1}\right)^{2} \lambda^{2}\right\} I_{0}^{2}(x)-2 u_{n, i} v^{2} I_{0}^{2}(x) .
$$

Summation of (18) and (19) gives

$$
-u_{n, i}\left\{v^{2} \Delta_{n} \lambda+\left(X_{i}-X_{i-1}\right)^{2} \lambda^{2}\right\}\left(2 I_{1}^{2}(x)-I_{0}(x) I_{2}(x)\right) .
$$

Putting together

$$
-u_{n, i}\left\{v^{2} \Delta_{n} \lambda+\left(X_{i}-X_{i-1}\right)^{2} \lambda^{2}\right\} I_{0}^{2}(x)
$$

and Eq. 21 it remains to study the sign of

$$
I_{0}^{2}(x)+2 I_{1}(x)^{2}-I_{0}(x) I_{2}(x)=I_{0}(x)\left(I_{0}(x)-I_{2}(x)\right)+2 I_{1}^{2}(x)
$$

which is positive due to the fact that $I_{0}(x)>I_{2}(x)$ for positive $x$ (from property (14)). This concludes the proof.

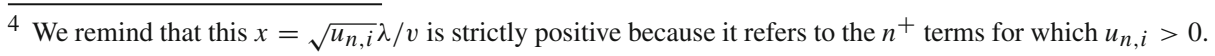


By previous theorem, it emerges that the contrast estimator $\hat{\lambda}_{n}$ is also a pseudo maximum likelihood estimator in the following sense

$$
\hat{\lambda}_{n}=\arg \max _{\lambda>0} L_{n}(\lambda) .
$$

Of course, $\hat{\lambda}_{n}$ is not a true maximum likelihood estimator as $L_{n}(\lambda)$ is not itself a true likelihood, nevertheless such estimators can be effective as in Kessler (2000). We now show that $\hat{\lambda}_{n}$ converges to the true maximum likelihood estimator of $\lambda$ in the case of continuous time observations of the telegraph process.

Theorem 2.2 Under the condition $n \Delta_{n}=T, \Delta_{n} \rightarrow 0$ as $n \rightarrow \infty$, we have that

$$
\hat{\lambda}_{n} \rightarrow \hat{\lambda}_{\infty}=\frac{N(T)}{T} .
$$

Proof In order to prove (23) we recall some properties of Bessel's functions (see e.g. Sect. 5.7, Lebedev (1972))

$$
\frac{\mathrm{d}}{\mathrm{d} x} I_{n}(x)=\frac{1}{2}\left(I_{n-1}(x)+I_{n+1}(x)\right)
$$

and

$$
\begin{aligned}
& \lim _{u \rightarrow 0} \frac{I_{1}(k \cdot u)}{u}=\frac{k}{2}, \quad \lim _{u \rightarrow 0} I_{0}(k \cdot u)=1, \\
& \lim _{u \rightarrow 0} I_{1}(k \cdot u)=0, \quad \lim _{u \rightarrow 0} I_{2}(k \cdot u)=0 .
\end{aligned}
$$

In the limit as $n \rightarrow \infty$ we have, $\Delta_{n} \rightarrow 0, n \Delta_{n}=T, u=u_{n, i}=v^{2} \Delta_{n}^{2}-\left(X_{i}-X_{i-1}\right)^{2} \rightarrow 0$ and $n^{+} \rightarrow N(T)$. Therefore (13) converges to

$$
-T+\frac{N(T)}{\lambda}
$$

which concludes the proof.

\subsection{A moment type estimator}

We already mentioned that the telegrapher's process is not stationary at the second order, but we can still think to use (7) to obtain a moment type estimator. If, as before, we consider the observations $X_{i}-X_{i-1}$ as $n$ copies of the process $X\left(\Delta_{n}\right)$, then looking at (7) for $t=\Delta_{n}$ we have

$$
E X^{2}\left(\Delta_{n}\right)=\frac{v^{2}}{\lambda}\left(\Delta_{n}-\frac{1-e^{-2 \lambda \Delta_{n}}}{2 \lambda}\right) .
$$

Consider now the sample second moment of the observed increments $X_{i}-X_{i-1}$

$$
m_{2}=\frac{1}{n} \sum_{i=1}^{n}\left(X_{i}-X_{i-1}\right)^{2}
$$

then the following estimator can be considered

$$
\tilde{\lambda}_{n}=\arg \min _{\lambda>0}\left\{m_{2}-\frac{v^{2}}{\lambda}\left(\Delta_{n}-\frac{1-e^{-2 \lambda \Delta_{n}}}{2 \lambda}\right)\right\}^{2} .
$$


The estimator $\tilde{\lambda}_{n}$ is also unique. Indeed, consider $V_{n}=m_{2} / \Delta_{n}^{2}$ which is an unbiased estimator of

$$
g_{n}(\lambda)=\frac{v^{2}}{\lambda \Delta_{n}^{2}}\left(\Delta_{n}-\frac{1-e^{-2 \lambda \Delta_{n}}}{2 \lambda}\right) .
$$

By Taylor's expansion we have that $g_{n}(\lambda) \simeq v^{2}-\frac{2}{3} v^{2} \lambda \Delta_{n}$, hence $g_{n}(\lambda)$ is a monotonic and decreasing function of $\lambda$ such that $\lim _{\lambda \rightarrow 0} g_{n}(\lambda)=v^{2}$ and $\lim _{\lambda \rightarrow \infty} g_{n}(\lambda)=0$. On the other hand $V_{n}$ is in $\left[0, v^{2}\right]$ because $0 \leq\left(X_{i}-X_{i-1}\right)^{2} \leq v^{2} \Delta_{n}^{2}$, hence $\tilde{\lambda}_{n}$ is the unique solution to (24).

Remark 2.1 We notice that for small $\Delta_{n}$, we have

$$
\frac{v^{2}}{\lambda}\left(\Delta_{n}-\frac{1-e^{-2 \lambda \Delta_{n}}}{2 \lambda}\right)=v^{2} \Delta_{n}^{2}-\frac{2}{3} v^{2} \lambda \Delta_{n}^{3}+o\left(\Delta_{n}^{3}\right)
$$

hence an approximate explicit solution of (24) is

$$
\tilde{\lambda}_{n}=\frac{3}{2}\left(\frac{1}{\Delta_{n}}-\frac{m_{2}}{v^{2} \Delta_{n}^{3}}\right)+o(1) .
$$

Properties of moment type estimators under the additional assumption $n \Delta_{n}^{3} \rightarrow 0$ and in the asymptotic framework $n \Delta_{n}=T \rightarrow \infty$ have been studied in Iacus and Yoshida (2006). Under the current scheme, i.e. when $n \Delta_{n}=T$ is fixed, we are only able to investigate numerically the properties of $\tilde{\lambda}_{n}$ for small sample sizes and compare it with the estimator $\hat{\lambda}_{n}$ (see Sect. 4).

\section{Parametric estimation for the geometric telegraph process}

Consider the process $Y$ of the observed log-returns

$$
Y_{i}=\log \frac{S_{i}}{S_{i-1}}=\alpha \Delta_{n}+\sigma\left(X_{i}-X_{i-1}\right)
$$

where $S_{i}=S\left(t_{i}\right)$ are discrete observations from the geometric telegraph process (4). We assume $\mu$ to be known, which is usually the case in finance where $\mu$ is related to the expected return of non risky assets like bonds, etc. The parameters $\sigma$ and $\lambda$ are to be estimated. As in the previous sections, we can assume $v$ to be known as well, if not we will show in the next paragraph a simple way to obtain it. We assume $Y_{i}$ to be $n$ copies of the process

$$
Y\left(\Delta_{n}\right)=\alpha \Delta_{n}+\sigma X\left(\Delta_{n}\right)
$$

with $X\left(\Delta_{n}\right)=X_{i}-X_{i-1}$ and $X(0)=x_{0}=0$. Therefore, by (6), we have

$$
\mathrm{E} Y\left(\Delta_{n}\right)=\alpha \Delta_{n}
$$

and by (7) we obtain

$$
\operatorname{Var} Y\left(\Delta_{n}\right)=\sigma^{2} \operatorname{Var} X\left(\Delta_{n}\right)=\sigma^{2} \frac{v^{2}}{\lambda}\left(\Delta_{n}-\frac{1-e^{-2 \lambda \Delta_{n}}}{2 \lambda}\right)
$$

A good estimator of the volatility $\sigma$ can be derived from the sample mean of the log returns. Indeed,

$$
\bar{Y}_{n}=\frac{1}{n} \sum_{i=1}^{n} Y_{i}=\alpha \Delta_{n}+\frac{\sigma}{n} X_{n}
$$


and

$$
\mathrm{E} \bar{Y}_{n}=\alpha \Delta_{n}+\frac{\sigma}{n} \mathrm{E} X_{n}=\alpha \Delta_{n}=\left(\mu-\frac{1}{2} \sigma^{2}\right) \Delta_{n}
$$

again by (6) and for the properties of the log-returns. From (27) we have that

$$
\sigma^{2}=2\left(\mu-\frac{\mathrm{E} \bar{Y}_{n}}{\Delta_{n}}\right)
$$

from which the following unbiased estimator of $\sigma^{2}$ can be derived

$$
\hat{\sigma}_{n}^{2}=2\left(\mu-\frac{\bar{Y}_{n}}{\Delta_{n}}\right) \text {. }
$$

Therefore, a reasonable moment type estimator of $\sigma$ is

$$
\hat{\sigma}_{n}=\sqrt{2\left(\mu-\frac{\bar{Y}_{n}}{\Delta_{n}}\right)}
$$

which not always exists because there is no guarantee that $\mu>\bar{Y}_{n} / \Delta_{n}$. Moreover, it should be noticed that in practice, given $\mu, \sigma$ and $\Delta_{n}$ the estimator essentially depends on the last value of the telegraph process $X_{n}$. In fact, we can write (28) in terms of the telegraph process

$$
\sqrt{2\left(\mu-\frac{\bar{Y}_{n}}{\Delta_{n}}\right)}=\sqrt{2\left(\mu-\alpha-\frac{\sigma}{T} X_{n}\right)} .
$$

We then use $\hat{\sigma}_{n}$ to estimate $\lambda$ making use of (25). Let

$$
\bar{s}_{Y}^{2}=\frac{1}{n} \sum_{i=1}^{n}\left(Y_{i}-\bar{Y}_{n}\right)^{2}
$$

then the proposed estimator of $\lambda$ is

$$
\dot{\lambda}_{n}=\arg \min _{\lambda>0}\left(\bar{s}_{Y}^{2}-\hat{\sigma}_{n}^{2} \frac{v^{2}}{\lambda}\left(\Delta_{n}-\frac{1-e^{-2 \lambda \Delta_{n}}}{2 \lambda}\right)\right)^{2} .
$$

Similar considerations of Remark 2.1 on the estimator $\tilde{\lambda}_{n}$ apply to $\dot{\lambda}_{n}$.

3.1 Filtering of the geometric telegraph process

If the velocity $v$ is not known one can proceed as follows: set

$$
Z_{i}=\frac{Y_{i}-\mathrm{E} \bar{Y}_{n}}{\sigma}=X_{i}-X_{i-1}=X\left(\Delta_{n}\right),
$$

an estimator of the increments of the telegraph process is

$$
\hat{Z}_{i}=\frac{Y_{i}-\bar{Y}_{n}}{\hat{\sigma}_{n}}=\hat{X}\left(\Delta_{n}\right), \quad i=1, \ldots, n .
$$

Then

$$
\hat{Z}_{1}=\hat{X}_{1}, \quad \hat{Z}_{2}+\hat{Z}_{1}=\hat{X}_{2}, \quad \hat{Z}_{3}+\hat{Z}_{2}+\hat{Z}_{1}=\hat{X}_{3}, \ldots
$$

where $\hat{X}_{i}$ are the estimated states of the underlying telegrapher's process. From these estimates, one can proceed as in previous sections and estimate both $\lambda$ and $v$. 
Table 1 Empirical performance of the minimum contrast estimator (22) for different values of the parameter $\lambda$ and different sample size

\begin{tabular}{|c|c|c|c|c|c|}
\hline$\lambda$ & Bias & $\sqrt{\operatorname{MSE}(\lambda)}$ & $\min \hat{\lambda}_{n}$ & $\max \hat{\lambda}_{n}$ & $n$ \\
\hline \multirow[t]{4}{*}{0.10} & -0.002 & 0.018 & 0.04 & 0.20 & 50 \\
\hline & -0.001 & 0.016 & 0.05 & 0.16 & 100 \\
\hline & -0.000 & 0.014 & 0.06 & 0.16 & 500 \\
\hline & -0.000 & 0.014 & 0.05 & 0.15 & 1000 \\
\hline \multirow[t]{4}{*}{0.25} & -0.011 & 0.041 & 0.13 & 0.47 & 50 \\
\hline & -0.003 & 0.031 & 0.16 & 0.41 & 100 \\
\hline & -0.000 & 0.023 & 0.16 & 0.34 & 500 \\
\hline & -0.000 & 0.023 & 0.16 & 0.35 & 1000 \\
\hline \multirow[t]{4}{*}{0.50} & -0.062 & 0.092 & 0.26 & 0.78 & 50 \\
\hline & -0.011 & 0.059 & 0.32 & 0.85 & 100 \\
\hline & -0.001 & 0.035 & 0.37 & 0.65 & 500 \\
\hline & -0.000 & 0.033 & 0.37 & 0.63 & 1000 \\
\hline \multirow[t]{4}{*}{0.75} & -0.151 & 0.175 & 0.36 & 1.01 & 50 \\
\hline & -0.031 & 0.091 & 0.47 & 1.18 & 100 \\
\hline & -0.001 & 0.048 & 0.58 & 0.96 & 500 \\
\hline & -0.000 & 0.043 & 0.60 & 0.92 & 1000 \\
\hline \multirow[t]{4}{*}{1.00} & -0.264 & 0.283 & 0.45 & 1.23 & 50 \\
\hline & -0.064 & 0.128 & 0.62 & 1.53 & 100 \\
\hline & -0.001 & 0.058 & 0.79 & 1.26 & 500 \\
\hline & -0.001 & 0.051 & 0.81 & 1.22 & 1000 \\
\hline \multirow[t]{4}{*}{1.50} & -0.546 & 0.558 & 0.58 & 1.48 & 50 \\
\hline & -0.162 & 0.227 & 0.90 & 2.16 & 100 \\
\hline & -0.003 & 0.080 & 1.24 & 1.86 & 500 \\
\hline & -0.001 & 0.066 & 1.27 & 1.77 & 1000 \\
\hline \multirow[t]{4}{*}{2.00} & -0.874 & 0.882 & 0.75 & 1.65 & 50 \\
\hline & -0.298 & 0.357 & 1.11 & 2.67 & 100 \\
\hline & -0.006 & 0.106 & 1.63 & 2.47 & 500 \\
\hline & -0.000 & 0.083 & 1.68 & 2.33 & 1000 \\
\hline
\end{tabular}

The time horizon $T$ is fixed to 500. Results over 10,000 Monte Carlo paths of the telegraph process. See text for more details

\section{Monte Carlo analysis}

To assess the properties of the estimators (22) and (24) for fixed $n<\infty$ we run extensive Monte Carlo analysis. We simulate 10000 trajectories of the telegrapher's process on the interval $[0, T], T=500$, for different values of $\lambda$ and with $v=1$ fixed. Each trajectory has then been resampled on a regular grid of $n=50,100,500$ and 1000 points and the corresponding observations have been used to estimate the unknown parameter. The results have been collected in Table 1. It emerges that, as expected, the bias tends asymptotically to zero as well as the mean square error. Furthermore, bias and variance are strictly correlated to the value of the unknown parameter $\lambda$. This is expected as well because, for fixed $n$, the more $\lambda$ increases the more Poisson events remain hidden to the observer. For the same experiment (and on the same sample trajectories), Table 2 reports the performance on the moment type estimator $\tilde{\lambda}_{n}$ from Eq. 24 .

Tables 3 and 4 reports estimates results on the geometric telegrapher's process respectively for the estimation of $\lambda$ and $\sigma$. The paths of the geometric telegrapher's process have been generated from the ones of the telegrapher process of Tables 1 and 2. As it can be seen, the estimator $\dot{\lambda}_{n}$ in Table 3 strongly depends on the quality of the estimate $\hat{\sigma}_{n}$ (reported in Table 4). For low values of $\lambda$, in some cases the condition for the existence of $\hat{\sigma}_{n}$, i.e. $\mu>\bar{Y}_{n} / \Delta_{n}$, 
Table 2 Empirical performance of the moment-type estimator (24) for different values of the parameter $\lambda$ and different sample size

\begin{tabular}{|c|c|c|c|c|c|}
\hline$\lambda$ & Bias & $\sqrt{\operatorname{MSE}(\lambda)}$ & $\min \tilde{\lambda}_{n}$ & $\max \tilde{\lambda}_{n}$ & $n$ \\
\hline \multirow[t]{4}{*}{0.10} & 0.002 & 0.022 & 0.04 & 0.24 & 50 \\
\hline & 0.001 & 0.018 & 0.05 & 0.18 & 100 \\
\hline & 0.000 & 0.016 & 0.05 & 0.16 & 500 \\
\hline & -0.000 & 0.016 & 0.05 & 0.16 & 1000 \\
\hline \multirow[t]{4}{*}{0.25} & 0.007 & 0.051 & 0.13 & 0.55 & 50 \\
\hline & 0.003 & 0.037 & 0.14 & 0.41 & 100 \\
\hline & 0.000 & 0.026 & 0.16 & 0.36 & 500 \\
\hline & 0.000 & 0.025 & 0.17 & 0.35 & 1000 \\
\hline \multirow[t]{4}{*}{0.50} & 0.018 & 0.106 & 0.25 & 1.13 & 50 \\
\hline & 0.007 & 0.070 & 0.32 & 0.93 & 100 \\
\hline & 0.001 & 0.040 & 0.35 & 0.66 & 500 \\
\hline & 0.000 & 0.037 & 0.35 & 0.65 & 1000 \\
\hline \multirow[t]{4}{*}{0.75} & 0.028 & 0.161 & 0.40 & 1.72 & 50 \\
\hline & 0.012 & 0.105 & 0.45 & 1.32 & 100 \\
\hline & 0.001 & 0.054 & 0.56 & 1.01 & 500 \\
\hline & 0.000 & 0.048 & 0.58 & 0.96 & 1000 \\
\hline \multirow[t]{4}{*}{1.00} & 0.040 & 0.219 & 0.53 & 2.42 & 50 \\
\hline & 0.017 & 0.141 & 0.62 & 1.80 & 100 \\
\hline & 0.002 & 0.066 & 0.73 & 1.27 & 500 \\
\hline & 0.001 & 0.057 & 0.77 & 1.25 & 1000 \\
\hline \multirow[t]{4}{*}{1.50} & 0.059 & 0.329 & 0.72 & 3.00 & 50 \\
\hline & 0.028 & 0.218 & 0.92 & 2.78 & 100 \\
\hline & 0.003 & 0.093 & 1.19 & 1.89 & 500 \\
\hline & 0.001 & 0.075 & 1.25 & 1.79 & 1000 \\
\hline \multirow[t]{4}{*}{2.00} & 0.080 & 0.412 & 1.05 & 3.00 & 50 \\
\hline & 0.035 & 0.290 & 1.22 & 3.00 & 100 \\
\hline & 0.006 & 0.120 & 1.59 & 2.46 & 500 \\
\hline & 0.003 & 0.095 & 1.66 & 2.39 & 1000 \\
\hline
\end{tabular}

The time horizon $T$ is fixed to 500. Results over 10,000 Monte Carlo paths of the telegraph process. See text for more details

has not been fulfilled hence we report the percentage of valid paths over the 10,000 replications. For $\lambda \geq 0.75$, it seems that $\dot{\lambda}_{n}$ performs quite similarly to $\tilde{\lambda}_{n}$ in terms of bias. This seems consistent with the definition of the estimators and the performance of the estimator $\hat{\sigma}_{n}$. Tables also report the column $\sqrt{\mathrm{MSE}}$. Values under this column are calculated, e.g. for the estimator $\hat{\lambda}_{n}$, as follows

$$
\sqrt{\operatorname{MSE}(\lambda)}=\sqrt{\frac{1}{N} \sum_{i=1}^{N}\left(\hat{\lambda}_{n}^{(i)}-\lambda\right)^{2}}
$$

where $N$ is the number of Monte Carlo simulations ( $N=10,000$ in our case) and $n$ is the fixed sample size. Notice that, by (29) the estimator $\hat{\sigma}_{n}^{2}$ depends on the observations only through $X_{n}$. Therefore, in our simulation scheme, because we sub-sample the observations $X_{0}, \ldots, X_{n}$ keeping fixed both $X_{0}$ and $X_{n}$, the estimator itself does not depend on the sample size $n$.

All the tables report the values $\min \hat{\theta}_{n}$ and $\max \hat{\theta}_{n}\left(\hat{\theta}_{n}\right.$ one among $\hat{\lambda}_{n}, \tilde{\lambda}_{n}, \dot{\lambda}_{n}$ and $\left.\hat{\sigma}_{n}^{2}\right)$ which represent the minimal and maximal value of the estimator over all Monte Carlo replications. 
Table 3 Empirical performance of the moment-type estimator (30) given the estimate $\hat{\sigma}_{n}$ (see Table 4), for different values of the parameter $\lambda$ and different sample size

\begin{tabular}{|c|c|c|c|c|c|c|}
\hline$\lambda$ & Bias & $\sqrt{\operatorname{MSE}(\lambda)}$ & $\min \dot{\lambda}_{n}$ & $\max \dot{\lambda}_{n}$ & $\%$ valid cases & $n$ \\
\hline \multirow[t]{4}{*}{0.10} & 0.018 & 0.107 & 0.00 & 1.01 & 96 & 50 \\
\hline & 0.042 & 0.157 & 0.00 & 1.12 & 96 & 100 \\
\hline & 0.301 & 0.609 & 0.00 & 3.40 & 96 & 500 \\
\hline & 0.634 & 1.189 & 0.00 & 6.48 & 96 & 1000 \\
\hline \multirow[t]{4}{*}{0.25} & 0.009 & 0.132 & 0.00 & 0.91 & 99 & 50 \\
\hline & 0.006 & 0.161 & 0.00 & 0.95 & 99 & 100 \\
\hline & 0.123 & 0.427 & 0.00 & 2.27 & 99 & 500 \\
\hline & 0.320 & 0.776 & 0.00 & 4.00 & 99 & 1000 \\
\hline \multirow[t]{4}{*}{0.50} & 0.022 & 0.185 & 0.00 & 1.64 & 100 & 50 \\
\hline & 0.010 & 0.186 & 0.00 & 1.27 & 100 & 100 \\
\hline & 0.031 & 0.395 & 0.00 & 2.03 & 100 & 500 \\
\hline & 0.139 & 0.635 & 0.00 & 3.33 & 100 & 1000 \\
\hline \multirow[t]{4}{*}{0.75} & 0.031 & 0.239 & 0.08 & 1.99 & 100 & 50 \\
\hline & 0.014 & 0.215 & 0.00 & 1.90 & 100 & 100 \\
\hline & 0.002 & 0.394 & 0.00 & 2.25 & 100 & 500 \\
\hline & 0.049 & 0.612 & 0.00 & 3.28 & 100 & 1000 \\
\hline \multirow[t]{4}{*}{1.00} & 0.045 & 0.304 & 0.31 & 2.68 & 100 & 50 \\
\hline & 0.021 & 0.256 & 0.26 & 2.24 & 100 & 100 \\
\hline & -0.000 & 0.388 & 0.00 & 2.65 & 100 & 500 \\
\hline & 0.012 & 0.608 & 0.00 & 3.69 & 100 & 1000 \\
\hline \multirow[t]{4}{*}{1.50} & 0.061 & 0.419 & 0.57 & 4.22 & 100 & 50 \\
\hline & 0.028 & 0.330 & 0.59 & 3.22 & 100 & 100 \\
\hline & -0.004 & 0.380 & 0.03 & 3.00 & 100 & 500 \\
\hline & -0.014 & 0.580 & 0.00 & 3.97 & 100 & 1000 \\
\hline \multirow[t]{4}{*}{2.00} & 0.094 & 0.529 & 0.80 & 6.49 & 100 & 50 \\
\hline & 0.038 & 0.402 & 0.93 & 4.16 & 100 & 100 \\
\hline & 0.005 & 0.380 & 0.64 & 3.46 & 100 & 500 \\
\hline & -0.002 & 0.536 & 0.00 & 4.08 & 100 & 1000 \\
\hline
\end{tabular}

The time horizon $T$ is fixed to 500. Results over 10,000 Monte Carlo paths of the geometric telegraph process. '\% valid cases' $=$ percentage of valid cases, i.e. simulated paths such that the estimator exists, i.e. simulated paths such that the estimator of $\sigma$ exists, i.e. $\mu>\bar{Y}_{n} / \Delta_{n}$. See text for more details

Table 4 Empirical performance of the estimator $\hat{\sigma}_{n}$ of (28) for different values of the parameter $\lambda$, for different sample size and $\sigma=0.5$

\begin{tabular}{lllllll}
\hline$\lambda$ & Bias & $\sqrt{\operatorname{MSE}(\sigma)}$ & $\min \hat{\sigma}_{n}$ & $\max \hat{\sigma}_{n}$ & \% valid cases & $n$ \\
\hline 0.10 & -0.008 & 0.138 & 0.01 & 0.85 & 96 & $50,100,500,1000$ \\
0.25 & -0.007 & 0.094 & 0.02 & 0.74 & 99 & $50,100,500,1000$ \\
0.50 & -0.003 & 0.065 & 0.09 & 0.69 & 100 & $50,100,500,1000$ \\
0.75 & -0.002 & 0.053 & 0.22 & 0.66 & 100 & $50,100,500,1000$ \\
1.00 & -0.002 & 0.045 & 0.30 & 0.66 & 100 & $50,100,500,1000$ \\
1.50 & -0.002 & 0.037 & 0.33 & 0.64 & 100 & $50,100,500,1000$ \\
2.00 & -0.001 & 0.031 & 0.36 & 0.61 & 100 & $50,100,500,1000$
\end{tabular}

The time horizon $T$ is fixed to 500. Results over 10,000 Monte Carlo paths of the geometric telegraph process. ' $\%$ valid cases' $=$ percentage of valid cases, i.e. simulated paths such that the estimator of $\sigma$ exists, i.e. $\mu>\bar{Y}_{n} / \Delta_{n}$. See text for more details 


\section{Conclusions}

Despite the analytical problems in dealing with the telegraph process, in this paper we have shown that statistical inference on the process can be attempted. Moreover, numerical results seem to suggest that also for the geometric telegraph process this attempt might be successful which encourages the study of the analytical properties of this process in view of financial applications. In particular, a detailed description of the law of the increments of the process of the log-returns might be of interest because, numerical evidence, show that these have heavy tails but their law do not need a high number of parameters like other distributions proposed in the literature (see e.g. Eberlain and Keller 1995). Moreover, the parameters have a direct interpretation as in the standard geometric Brownian motion. This will be a topic for future research.

Acknowledgements We thank an anonymous referee for his/her useful remarks and comments on the present work. This work has been supported by the Italian Ministry of University and Research, grant F.I.R.B. num. RBNE03E3KF_004

\section{References}

Black F, Scholes MS (1973) The pricing of options and corporate liabilities. J Polit Econ 81:637-654

Di Crescenzo A, Pellerey F (2002) On prices' evolutions based on geometric telegrapher's process. Appl Stochastic Models Bus Ind 18:171-184

Di Masi GB, Kabanov Yu M, Runggaldier WJ (1994) Mean-variance hedging of options on stocks with Markov volatilities. Theor Probab Appl 39:172-182

Eberlain E, Keller U (1995) Hyperbolic distributions in finance. Bernoulli 1:281-299

Florens-Zmirou D (1989) Approximate discrete time schemes for statistics of diffusion processes. Statistics 20:547-557

Foong SK, Kanno S (1994) Properties of the telegrapher's random process with or without a trap. Stochastic Processes Appl 53:147-173

Goldstein S (1951) On diffusion by discontinuous movements and the telegraph equation. Q J Mech Appl Math 4:129-156

Iacus SM (2001) Statistic analysis of the inhomogeneous telegrapher's process. Stat Prob Lett 55(1):83-88

Iacus SM, Yoshida N (2006) Estimation for the discretely observed telegraph process. Dec. 2006. available at http://services.bepress.com/unimi/statistics/art21/

Kac M (1974) A stochastic model related to the telegrapher's equation. Rocky Mountain J Math 4:497-509

Kessler M (2000) Simple and explicit estimating functions for discretely observed diffusion process. Scand J Stat 27:65-82

Kutoyants Yu A (1998) Statistical inference for spatial Poisson processes. Lecture Notes in Statistics, SpringerVerlag

Le Breton A (1976) On continuous and discrete sampling for parameter estimation in diffusion type processes. Math Program Study 5:124-144

Lebedev NN (1972) Special functions and their applications. Dover Publications, Inc., New York

Mazza C, Rulliére D (2004) A link between wave governed random motions and ruin processes. Insur Math Econ 35:205-222

Merton RC (1973) Theory of rational option pricing. Bell J Econ Manage Sci 4(1):141-183

Orsingher E (1990) Probability law, flow function, maximun distribution of wave-governed random motions and their connections with Kirchoff's laws. Stochastic Process Appl 34:49-66

Orsingher E (1995) Motions with reflecting and absorbing barriers driven by the telegraph equation. Random Oper Stochastic Equations 3:9-21

Ratanov N (2004) A jump telegraph model for option pricing. Borradores de Investigatión, 58, Nov. 2004, to appear on Quant Fin, available at http://economia.urosario.edu.co/investigacion/bi/bi58.pdf

Ratanov N (2005) Quantile hedging for telegraph markets and its applications to a pricing of equity-linked life insurance contracts. Borradores de Investigatión, 62. Apr. 2005, http://www.urosario.edu.co/FASE1/ economia/documentos/pdf/bi62.pdf

Sørensen M (2000) Prediction-based estimating functions. Econ J 3:123-147 
Sørensen H (2004) Parametric inference for diffusion processes observed at discrete points time: a survey. Int Stat Rev 72:337-354

Stadje W, Zacks S (2004) Telegraph processes with random velocities. J Appl Prob 41:665-678

Yao Y (1985) Estimation of noisy telegraph process: nonlinear filtering versus nonlinear smoothing. IEEE Trans Inf Theor 31(3):444-446 